\title{
Q2 Contribution of genetic and epigenetic mechanisms to Wnt pathway activity in prevalent skeletal disorders
}

\author{
Q1 Carmen García-Ibarbia a , Jesús Delgado-Calle a , Iñigo Casafont ${ }^{\text {b }}$, Javier Velasco a , Jana Arozamena a , \\ María I. Pérez-Núñez ${ }^{c}$, María A. Alonso ${ }^{c}$, María T. Berciano ${ }^{b}$, Fernando Ortiz ${ }^{a}$, José L. Pérez-Castrillón ${ }^{\mathrm{d}}$, \\ Agustín F. Fernández ${ }^{\mathrm{e}}$, Mario F. Fraga ${ }^{\mathrm{e}}$, María T. Zarrabeitia ${ }^{\mathrm{f}}$, José A. Riancho ${ }^{\mathrm{a}, *}$ \\ a Department of Internal Medicine, Hospital U.M. Valdecilla-IFIMAV, University of Cantabria, RETICEF, Santander, Spain \\ b Department of Anatomy and Cell Biology, University of Cantabria, IFIMAV, Santander, Spain \\ c Department of Orthopaedic Surgery and Traumatology, Hospital U.M. Valdecilla, Santander, Spain \\ d Department of Internal Medicine, Hospital U, Rio-Hortega, University of Valladolid, Valladolid, Spain \\ e Cancer Epigenetics Laboratory, Instituto Universitario de Oncología del Principado de Asturias (IUOPA), HUCA, University of Oviedo, Oviedo, Spain \\ ${ }^{\mathrm{f}}$ Unit of Legal Medicine, University of Cantabria, IFIMAV, Santander, Spain
}

\section{A R T I C L E I N F O}

Article history:

Accepted 23 September 2013

Available online $\mathrm{xxxx}$

\section{Keywords:}

Wnt

$\beta$-Catenin

DNA methylation

Fractures

Bone diseases

\section{A B S T R A C T}

We reported previously that the expression of Wnt-related genes is lower in osteoporotic hip fractures than in 26 osteoarthritis. We aimed to confirm those results by analyzing $\beta$-catenin levels and explored potential genetic 27 and epigenetic mechanisms involved.

$\beta$-Catenin gene expression and nuclear levels were analyzed by real time PCR and confocal immunofluorescence. 29 Increased nuclear $\beta$-catenin was found in osteoblasts isolated from patients with osteoarthritis (99 \pm 430 units vs. $76 \pm 12, \mathrm{p}=0.01, \mathrm{n}=10$ ), without differences in gene transcription, which is consistent with 31 a post-translational down-regulation of $\beta$-catenin and decreased Wnt pathway activity.

Twenty four single nucleotide polymorphisms (SNPs) of genes showing differential expression between fractures 33 and osteoarthritis (WNT4, WNT10A, WNT16 and SFRP1) were analyzed in DNA isolated from blood of 853 pa- 34 tients. The genotypic frequencies were similar in both groups of patients, with no significant differences. 35 Methylation of Wnt pathway genes was analyzed in bone tissue samples (15 with fractures and 15 with osteo- 36 arthritis) by interrogating a CpG-based methylation array. Six genes showed significant methylation differences 37 between both groups of patients: FZD10, TBL1X, CSNK1E, WNT8A, CSNK1A1L and SFRP4. The DNA demethylating 38 agent 5-deoxycytidine up-regulated 8 genes, including FZD10, in an osteoblast-like cell line, whereas it down- 39 regulated other 16 genes.

In conclusion, Wnt activity is reduced in patients with hip fractures, in comparison with those with osteoarthritis. 41 It does not appear to be related to differences in the allele frequencies of the Wnt genes studied. On the other 42 hand, methylation differences between both groups could contribute to explain the differences in Wnt activity. 43

(C) 2013 Published by Elsevier B.V. 44
Abbreviations: AzadC, 5-aza-2-deoxy-azacytidine; CACYBP, calcyclin binding protein CAMK2G, calcium/calmodulin-dependent protein kinase II gamma; CSNK1A1, casein kinase 1, alpha 1; CSNK1A1L, casein kinase 1, alpha 1-like; CSNK1E, casein kinase 1, epsilon; $\mathrm{Ct}$, threshold cycle; CTBP1, C-terminal binding protein 1; FDR, false discovery rate; FOSL1, FOS-like antigen 1; FRZB, frizzled-related protein; FZD10, frizzled homolog 10; GSK3B, glycogen synthase kinase 3 beta; GWAS, genome-wide association study; HWE, HardyWeinberg equilibrium; LRP5, lipoprotein receptor related protein 5; PLCB3, phospholipase C, beta 3 (phosphatidylinositol-specific); PPP2R1A, protein phosphatase 2 (formerly 2A), regulatory subunit A, alpha isoform; RHOA, ras homolog gene family, member A; SFRP1, secreted frizzled-related protein 1; SFRP4, secreted frizzled-related protein 4; TBL1X, transducin (beta)-like 1X-linked; TBP, TATA box binding protein; WNT10A, winglesstype MMTV integration site family, member 10A; WNT16, wingless-type MMTV integration site family, member 16; WNT4, wingless-type MMTV integration site family, member 4; WNT8A, wingless-type MMTV integration site family, member 8A.

* Corresponding author at: Department of Internal Medicine, Hospital U.M. Valdecilla, University of Cantabria, IFIMAV, Santander 39008, Spain. Tel.: + 34 942201990; fax: + 34942201695

E-mail address: rianchoj@unican.es (J.A. Riancho).

\section{Introduction}

The Wnt pathway has emerged as an important regulator of skeletal 50 homeostasis. Binding of Wnt ligands to their receptors triggers the acti- 51 vation of a complex signaling pathway. Multiple intracellular mediators 52 are involved, but the best known cascade of Wnt signals constitutes the 53 so-called canonical pathway, which involves the post-transcriptional 54 regulation of $\beta$-catenin levels. Wnt ligands induce the disassembly 55 of the GSK3 complex that phosphorylates $\beta$-catenin. Since non- 56 phosphorylated $\beta$-catenin is less prone to proteasome degradation, 57 this results in increased $\beta$-catenin levels and translocation into the 58 nucleus, where it modulates the transcription of target genes (Gaur 59 et al., 2005; Williams and Insogna, 2009), with the collaboration of 60 several co-factors, including members of the T-cell factor/lymphoid 61 enhancing factor family (Gordon and Nusse, 2006; MacDonald et al., 62 2009). There are 19 different Wnt ligands, some of which, such as 63 
Wnt3a, preferentially activate the canonical pathway, whereas others, such as Wnt4 and Wnt5a, are usually regarded to transmit signals through non-canonical pathways. However, there is no a clear difference between ligands, each one being able to activate preferentially the canonical or non-canonical pathways depending on the target cell and other context-dependent factors (van Amerongen and Nusse, 2009). Furthermore, cross-talks take place between different pathways. Both the canonical and the non-canonical pathways appear to be involved in the regulation of bone homeostasis (Chang et al., 2007; Gaur et al., 2005; Piters et al., 2008). Wnt inhibitors include members of the secreted frizzled related protein family, which are structurally related to the Wnt membrane-bound receptors frizzled and bind directly to Wnt ligands, thereby altering their ability to interact with the Wnt receptor complex at cell membranes (Kawano and Kypta, 2003). One member of this family, sFRP1, has been shown to modulate the activity of cells of the osteoblastic lineage (Bodine et al., 2005; Yao et al., 2010). In line with this, knock-out mice with deletion of the SFRP1 or other genes encoding secreted frizzled proteins show increased bone mass (Lodewyckx and Lories, 2009).

An association between Wnt-related genes, particularly FRZB, with osteoarthritis was reported in several studies (Loughlin et al., 2004; Valdes et al., 2007), but it could not be confirmed in recent GWAS (Arcogen Consortium, 2012; Panoutsopoulou et al., 2011). On the other hand, variations in genes related to the Wnt pathway, such as the Wnt co-receptors LRP5 and the Wnt inhibitor sclerostin, have been related to osteoporosis in genetic association studies (Ralston, 2010; Riancho et al., 2011; Richards et al., 2009; Styrkarsdottir et al., 2009; Valero et al., 2011). Specifically, Estrada et al. found an association between some polymorphisms of genes encoding Wnt ligands, such as WNT16, and osteoporosis in a large multinational GWAS (Estrada et al., 2012).

Epigenetic mechanisms, and specifically the methylation of $\mathrm{CpG}$ sites in gene promoters, are known to play an important role in gene expression regulation during development and in adult organisms (Fraga and Esteller, 2007). The role of DNA methylation in bone homeostasis has not been extensively studied yet, but several lines of evidence point it as a critical regulator of the differentiation of bone cells (Delgado-Calle et al., 2012a,c).

Osteoporosis and osteoarthritis are prevalent skeletal disorders. Whereas bone mass is decreased in osteoporosis, several epidemiological studies suggested that patients with osteoarthritis may show not only periarticular bone formation, but also a generalized trend for higher bone mass (Arokoski et al., 2002; Chaganti et al., 2010; Dequeker et al., 2003). We have previously reported that, in comparison with patients with osteoarthritis, the expression of a number of genes in the Wnt pathway is reduced in bone samples and osteoblast cultures from patients with osteoporotic hip fractures. In line with this, experiments with a reporter vector suggested higher Wnt activity is osteoarthritis, suggesting that differences in Wnt activity may be involved in the opposite changes in bone mass typical of these disorders (Velasco et al., 2010). In the present study we compared nuclear $\beta$-catenin levels in primary osteoblasts from patients with hip fractures and hip osteoarthritis, determined $\beta$-catenin gene expression, and explored if the differences in Wnt activity were related to genotypic or epigenetic differences between both groups of patients.

\section{Materials and methods}

\subsection{Patients}

The study subjects included patients with osteoporotic hip fractures or with severe hip osteoarthritis requiring replacement surgery. Samples from different patients were used for the various experiments. Patients with secondary osteoarthritis or secondary osteoporosis, those taking drugs known to affect bone metabolism, as well as those having 126 fractures related to high-energy trauma, were excluded. 127

Patients gave informed consent. The study was approved by the 128 institutional review board (Comité de Etica en Investigación Clínica 129 de Cantabria).

\subsection{Osteoblast cultures}

131

Bone samples were obtained during hip replacement surgery, in pa- 132 tients with hip fractures $(n=11)$ or with hip osteoarthritis $(n=9) 133$ (mean age $82 \pm 5$ and $75 \pm 7 \mathrm{yr}$, respectively). Trabecular bone cylin- 134 ders of the central part of the femoral head (thus avoiding the fractured 135 and the subchondral regions) were obtained with a trephine, cut in 136 small samples, washed extensively in phosphate-buffered saline and 137 used to set up osteoblast cultures by the primary explant technique 138 (Jonsson et al., 1999). In brief, bone fragments were seeded into T-75 139 plastic flasks containing Dulbecco's modified Eagle's medium, antibi- 140 otics and 10\% fetal bovine serum. This allowed osteoblastic precursor 141 cells to migrate from the fragments and proliferate. After confluence, 142 cells were trypsinized and cultured in the appropriate experimental 143 conditions.

\subsection{Immunofluorescence microscopy}

145

Primary osteoblasts obtained from 5 patients with fractures and 4146 with osteoarthritis were grown on microscope glass coverslips until 147 $70-80 \%$ confluence. Then they were fixed with 3.7\% paraformaldehyde- 148 PBS and permeabilized with $0.5 \%$ Triton 100 -X for 30 min at room 149 temperature. After several washes with PBS and 0.05\% PBS-Tween, the 150 coverslips were incubated overnight at $4{ }^{\circ} \mathrm{C}$ with a rabbit polyclonal 151 anti $\beta$-catenin antibody (Abcam, Cambridge, UK), diluted 1/200 in PBS. 152 After washing, cells were incubated with a FITC-conjugated anti-rabbit 153 secondary antibody (Jackson ImmunoResearch Laboratories, West 154 Grove, PA, USA) for $45 \mathrm{~min}$ and mounted with VectaShield (Vector 155 Laboratories, Burlingame, CA, USA). In some experiments, $\beta$-catenin 156 immunolabeling was combined with Texas Red-Phalloidin to stain 157 actin filaments.

158

Confocal microscopy was performed with a laser scanning microscope 159 (LSM 510; Carl Zeiss, Oberkochen, Germany) by using excitation wave- 160 lengths of $488 \mathrm{~nm}$ (for FITC) and $543 \mathrm{~nm}$ (for Texas Red). Each channel 161 was recorded independently, and pseudocolor images were generated 162 and superimposed. TIFF images were transferred to Adobe Photoshop 163 7.0 software (Adobe Systems, San Jose, CA, USA) for presentation. 164

To measure fluorescence intensities of nuclear and cytoplasmic $\beta-165$ catenin in primary osteoblast cultures from patients with fractures 166 and osteoarthritis (blindly to the origin of the sample), confocal images 167 of at least 130 osteoblasts (without prior selection) of each patient 168 group were captured by using a 63× oil 1.4 (NA) objective. Images 169 were acquired with no saturated pixels, always using the same confocal 170 settings, with eightfold averaging at resolution of $1024 \times 1024$ pixels 171 and using a pinhole setting of 1 . In order to minimize between-day var- 172 iability bias, in each experiment cells of both patient groups were 173 included. Images were background corrected by reference regions out- 174 side the cells and fluorescence intensities were estimated by using the 175 ImageJ software (NIH, Bethesda, Maryland, USA; http://rsb.info.nih. 176 gov/ij/). Fluorescence intensities were measured in four regions of in- 177 terest of the same area per nucleus, excluding the nucleolus, and in 178 four regions per cytoplasm. Nuclear and cytoplasmic $\beta$-catenin average 179 values and the nucleocytoplasmic ratios were computed for each cell 180 analyzed and pooled for each patient. The mean values of each patient 181 group were then compared by two-tailed unpaired t-test.

\section{4. $\beta$-Catenin expression}

183

Sub-confluent cultures of first-passage primary osteoblasts were 184 used to analyze gene expression by reverse transcription (RT) real- 185 
time PCR. The medium was aspirated and fresh medium with $0.1 \%$ bovine serum albumin was added. Forty eight hours later, cells were rinsed with phosphate-buffered saline and the RNA was extracted with Trizol, following manufacturer's instructions (Invitrogen, Carlsbad, CA, USA). The purity and integrity of RNA were checked by absorbance and gel electrophoresis.

Aliquots of RNA were reverse-transcribed with the Superscript III kit (Invitrogen), using random hexamers as primers. After RT, the expression of $\beta$-catenin was determined by real-time PCR using genespecific primers and Taqman probes (Applied Biosystems, Foster City, CA, USA) in an ABI7300 apparatus (Applied Biosystems). The amount of PCR product was monitored by fluorescence and the threshold cycle (Ct) for each well was determined. The results were normalized to the expression of the housekeeping gene TATA box protein (TBP) and the specific gene expression was calculated as the $2^{-\Delta \mathrm{Ct}}$, where $\Delta \mathrm{Ct}$ is the difference between the gene of interest threshold cycle and the housekeeping threshold cycle.

\subsection{Genetic analysis}

The genotype analysis included 353 patients with severe hip osteoarthritis requiring replacement surgery (182 men and 171 women, age $69 \pm 11 \mathrm{yr}$ ) and 500 with hip fractures (92 men and 408 women, age $79 \pm 12 \mathrm{yr}$ ). DNA was isolated from blood or buccal swabs using a commercial kit (Qiagen, Hilden, Germany) and quantified with Qubit technique (Invitrogen). Several candidate genes of the Wnt pathway were selected on the basis that they showed differential expression in hip osteoarthritis and fractures (Velasco et al., 2010). The gene set included 3 Wnt ligands (WNT4, WNT10A and WNT16) and 1 gene encoding a soluble Wnt-binding protein of the frizzled family (SFRP4). We explored the Hapmap database searching SNPs in those genes with a minimum allelic frequency (MAF) of $10 \%$ in the Caucasian population. Tagging SNPs capturing the most common variants of these genes were then selected using the method of Gabriel, implemented in Haploview (Barrett et al., 2005). In addition, we included potentially functional SNPs identified with the PupaSuite web tool (Conde et al., 2006). The SNP set was genotyped using a Sequenom platform, at the Centro Nacional de Genotipado in Santiago de Compostela, Spain. Replicate samples were included for quality control.

\subsection{DNA methylation profiling}

Bone samples were obtained from femoral heads removed during hip replacement as previously reported (Hernandez et al., 2008). The study population included women aged $61-85$ years; with osteoarthritis (age $73 \pm 7 \mathrm{yr} ; \mathrm{n}=15$ ) or with osteoporotic hip fractures ( $81 \pm$ $3 \mathrm{yr} ; \mathrm{n}=15$ ). DNA was isolated from bone samples using phenol:chloroform:isoamylalcohol, as previously described (Delgado-Calle et al., 2012c). After bisulphite conversion of DNA using the EZ DNA Methylation kit (Zymo Research, Orange, CA, USA), microarray-based DNA methylation profiling was performed with the Human Methylation Infinium 27 k DNA Analysis BeadChip (Illumina, San Diego, CA, USA), following manufacturer's instructions. This array targets $\mathrm{CpG}$ sites located within the proximal promoter regions of transcription start sites of 14,475 consensus coding sequencing (CCDS) in the NCBI Database (Genome Build 36) and 110 miRNA promoters. The assay interrogates the loci using two site-specific probes, one designed for the methylated locus ( $M$ bead type) and another for the unmethylated locus ( $U$ bead type). The methylation level for the interrogated locus is determined by calculating the ratio of the fluorescent signals from the methylated vs. unmethylated sites as expressed as beta-values, a quantitative measure of DNA methylation levels of specific $\mathrm{CpG}$ that ranges from 0 for completely unmethylated to 1 for completely methylated. The analysis included $257 \mathrm{CpG}$ sites present in genes related to the Wnt pathway.

\subsection{Gene demethylation and expression}

To explore the effects of DNA demethylation on gene expression we 247 treated the osteoblast-like cell line MG-63 with 5-aza-2-deoxy- 248 azacytidine (AzadC) for 4 days. Three independent cultures (each 249 including control and $1 \mu \mathrm{M}$ AzadC-treated cells) were used for 250 these experiments. Gene expression profiling was performed at 251 the Gene Expression Unit of Genomics Core Facility (SGIKer) of the 252 University of the Basque Country UPV/EHU (Leioa, Spain) using 253 oligonucleotide-based Agilent Whole Human Genome Oligo Micro- 254 arrays $4 \times 44 \mathrm{~K}$ G4112F (design ID 014850). Two-color microarray- 255 based gene expression analysis was performed following the Quick 256 Amp Labeling protocol from Agilent Technologies (G4140-90050 257 v5.7; Agilent Technologies España, Las Rozas, Spain). In brief, 258 Cyanine-3 (Сy3) labeled cRNA was prepared from 500 ng of a pool 259 of control RNA samples, and Cyanine-5 (Cy5) labeled cRNA was pre- 260 pared from $300 \mathrm{ng}$ of individual AzadC-treated samples. Dye incor- 261 poration and cRNA yield were monitored with the NanoDrop ND- 262 1000 Spectrophotometer. Aliquots (825 ng) of each Cy3 and Cy5 263 labeled cRNA were fragmented, and co-hybridized to microarrays 264 and washed following manufacturer's recommendations. Slides 265 were scanned using an Agilent DNA Microarray Scanner G2565BA 266 and the resulting TIFF images were processed with Agilent Feature 267 Extraction Software v9.5.3. Default parameters for two-color gene 268 expression microarrays were used for image analysis, data extrac- 269 tion, background correction and dye bias correction. Non-uniform 270 outliers or signals not significantly above background intensity in 271 $70 \%$ or more of each channel (Cy3 or Cy5) were filtered out.

\subsection{Statistical analyses}

The statistical significance of the differences in $\beta$-catenin levels be- 274 tween samples from patients with hip fractures and osteoarthritis was 275 tested by the Mann-Whitney tests. Genotype and allele frequencies of 276 SNPs were tested for consistency with Hardy-Weinberg equilibrium 277 (HWE) proportions using Plink software (Purcell et al., 2007). The allelic 278 frequency distributions in both groups of patients were compared 279 assuming additive models with Plink, in the whole population and in 280 the male and females subgroups. Power analysis of the genetic analyses 281 was done assuming a log-additive genetic model with Quanto software 282 (available at http://hydra.usc.edu/gxe/).

For methylation, the ratio methylated/unmethylated was estimated 284 as the beta/(1-beta) ratio and log2-transformed. Data were then nor- 285 malized by the quintile procedure with BRB Array software developed 286 by Dr. Richard Simon and the BRB-ArrayTools Development Team 287 (http://linus.nci.nih.gov/BRB-ArrayTools.html) and between group dif- 288 ferences in the methylation of genes related to the Wnt pathway in 289 KEGG (Kyoto Encyclopedia of Genes and Genomes) were analyzed by 290 t-tests. Significance levels were corrected for multiple testing by the 291 method of Benjamini to control the FDR (Benjamini and Yekutieli, 292 2005).

To assess the AzadC-induced changes in gene expression, we consid- 294 ered as up-regulated those genes consistently increased in the three 295 experiments, with an average fold-change $>2$; similarly, consistently 296 decreased genes with a fold-change $<-2$ were regarded as down- 297 regulated.

\section{Results}

\section{1. $\beta$-Catenin levels and gene expression}

In addition to a weak immunostaining throughout the cytoplasm, 301 $\beta$-catenin signal was concentrated at the cell cortex, particularly be- 302 neath membrane domains involved in cell-cell interactions. In this 303 localization, $\beta$-catenin frequently colocalized with the tip of actin fil- 304 aments (Fig. 1). Staining was also found at the nuclei of cells of both 305 



Fig. 1. Immunofluorescence of primary osteoblasts grown from fracture (1) or osteoarthritis samples (2). A, $\beta$-Catenin staining; B, phalloidin staining of F-actin; C, merged images.

groups of patients, but the nuclear fluorescence intensity was significantly lower in cultures grown from patients with hip fractures than in patients with osteoarthritis (nucleocytoplasmatic ratios of $2.39 \pm 0.38$ versus $3.13 \pm 0.38$, respectively, $\mathrm{p}=0.023$ ). To analyze if $\beta$-catenin was regulated at transcriptional or post-transcriptional levels, we measured its expression in cell cultures. The abundance of $\beta$-catenin mRNA was similar in both groups of patients (Fig. 2).

\subsection{Association between gene polymorphisms and disease}

The SNPs analyzed and their chromosomal locations are shown in Supplementary Table S1. The genotype distribution did not differ between both groups of individuals (Table 1), neither in the combined analysis nor in the sex-stratified analysis. Similar results were obtained when age was included in the analysis as a covariate.

\subsection{DNA methylation and gene expression}

The analyzed CpG sites and their statistical significance are listed in 320 Table S2 (Supplementary Online Material). Statistical analysis revealed 321 6 CpG sites (out of the 257 related to Wnt pathway genes) with differ- 322 ential methylation in samples from patients with fractures and osteoar- 323 thritis (FDR < 0.1). They included FZD10, CSNK1E, TBL1X, WNT8A, and 324 SFRP4 genes. P-values and the differences in methylation are shown in 325 Table 2. The heatmap of the probe methylation, shown in Fig. 3, revealed 326 important heterogeneity among patients with fractures, who tended to 327 group into two different classes. Within the age range studied, no gene 328 showed significant age-related differences in methylation.

329

To explore the potential relationship between DNA methylation 330 and the expression of Wnt-related genes, we treated osteoblast- 331 like MG-63 cells with AzadC, which promotes a global decrease in 332
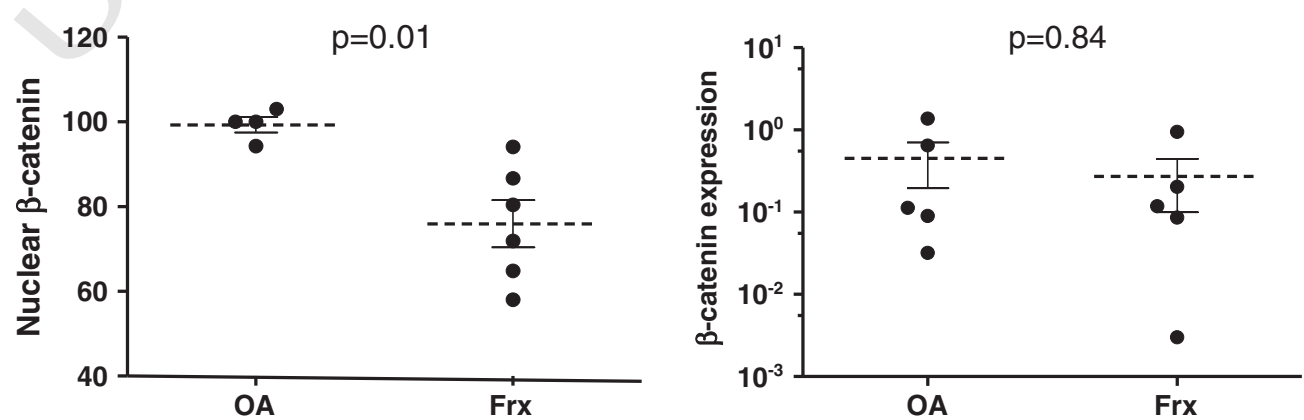

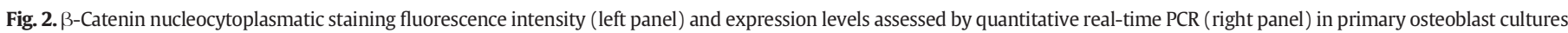
from patients with fractures (Frx) or osteoarthritis (OA). Each point represents the result of an individual patient. 
t1.4

$\mathrm{t} 1.5$

$\mathrm{t} 1.6$

t1.7

$\mathrm{t} 1.8$

t1.9

t1.10

$\mathrm{t} 1.11$

t1.12

t1.13

$\mathrm{t} 1.14$

t1.15

t1.16

t1.17

t1.18

$\mathrm{t} 1.19$

$\mathrm{t} 1.20$

$\mathrm{t} 1.21$

$\mathrm{t} 1.22$

t1. 23

$\mathrm{t} 1.24$

$\mathrm{t} 1.25$

t1. 26

$\mathrm{t} 1.27$

t1.28

$\mathrm{t} 1.29$

Table 1

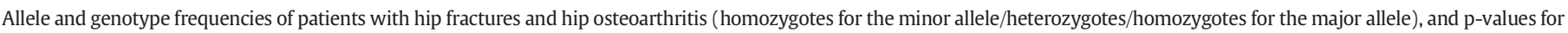
the differences between both groups of patients.

\begin{tabular}{|c|c|c|c|c|c|c|c|c|}
\hline \multirow[b]{2}{*}{ SNP } & \multirow[b]{2}{*}{ GENE } & \multicolumn{2}{|c|}{ Fracture $(n=500)$} & \multicolumn{2}{|c|}{ Osteoarthritis $(\mathrm{n}=353)$} & \multirow[b]{2}{*}{$\mathrm{P}($ all $)$} & \multirow[b]{2}{*}{$\mathrm{P}$ (women) } & \multirow[b]{2}{*}{$\mathrm{P}$ (men) } \\
\hline & & MAF & Genotypes & MAF & Genotypes & & & \\
\hline rs7526484 & WNT4 & 0.23 & $26 / 160 / 284$ & 0.24 & 18/123/193 & 0.56 & 0.46 & 0.98 \\
\hline rs2235526 & WNT4 & 0.18 & $17 / 130 / 303$ & 0.18 & $13 / 93 / 217$ & 0.92 & 0.39 & 0.65 \\
\hline rs10917158 & WNT4 & 0.18 & $17 / 133 / 321$ & 0.17 & $12 / 90 / 234$ & 0.70 & 0.91 & 0.51 \\
\hline rs3806557 & WNT10A & 0.23 & $30 / 159 / 286$ & 0.20 & $16 / 108 / 222$ & 0.18 & 0.27 & 0.83 \\
\hline rs10177996 & WNT10A & 0.21 & $27 / 136 / 280$ & 0.19 & $8 / 103 / 208$ & 0.19 & 0.15 & 0.77 \\
\hline rs2385199 & WNT10A & 0.20 & $24 / 149 / 309$ & 0.17 & $8 / 104 / 231$ & 0.14 & 0.16 & 0.89 \\
\hline rs3779381 & WNT16 & 0.26 & $34 / 174 / 258$ & 0.26 & $22 / 133 / 183$ & 0.92 & 0.88 & 0.33 \\
\hline rs2908004 & WNT16 & 0.44 & $87 / 252 / 144$ & 0.42 & $62 / 168 / 115$ & 0.46 & 0.76 & 0.94 \\
\hline rs2707471 & WNT16 & 0.16 & $13 / 122 / 338$ & 0.15 & $7 / 87 / 247$ & 0.65 & 0.90 & 0.91 \\
\hline rs3801385 & WNT16 & 0.07 & $3 / 58 / 412$ & 0.07 & $4 / 40 / 297$ & 0.83 & 0.65 & 0.94 \\
\hline rs2707466 & WNT16 & 0.44 & $87 / 241 / 142$ & 0.42 & $63 / 153 / 117$ & 0.37 & 0.53 & 0.92 \\
\hline rs17143305 & WNT16 & 0.15 & $12 / 120 / 335$ & 0.14 & $7 / 82 / 244$ & 0.58 & 0.89 & 0.90 \\
\hline rs3242 & SFRP1 & 0.38 & $77 / 192 / 183$ & 0.36 & $46 / 153 / 138$ & 0.45 & 0.88 & 0.86 \\
\hline rs1127379 & SFRP1 & 0.44 & $104 / 229 / 159$ & 0.44 & $64 / 175 / 106$ & 0.84 & 0.66 & 0.95 \\
\hline rs7820647 & SFRP1 & 0.39 & $76 / 212 / 173$ & 0.41 & $53 / 163 / 113$ & 0.58 & 0.31 & 0.73 \\
\hline rs11786592 & SFRP1 & 0.35 & $59 / 211 / 206$ & 0.36 & $44 / 145 / 139$ & 0.70 & 0.54 & 0.83 \\
\hline rs6651363 & SFRP1 & 0.40 & $77 / 225 / 177$ & 0.42 & $61 / 161 / 114$ & 0.31 & 0.58 & 0.33 \\
\hline rs10109536 & SFRP1 & 0.41 & $83 / 233 / 176$ & 0.42 & $66 / 159 / 124$ & 0.64 & 0.42 & 0.98 \\
\hline rs17652488 & SFRP1 & 0.51 & $127 / 189 / 121$ & 0.51 & $94 / 130 / 86$ & 0.83 & 0.62 & 0.84 \\
\hline rs10958671 & SFRP1 & 0.13 & $13 / 98 / 377$ & 0.13 & $6 / 75 / 259$ & 0.96 & 0.50 & 0.86 \\
\hline rs 17574424 & SFRP1 & 0.19 & $22 / 145 / 321$ & 0.16 & $6 / 99 / 240$ & 0.09 & 0.15 & 0.27 \\
\hline rs7832767 & SFRP1 & 0.05 & $2 / 45 / 425$ & 0.05 & $1 / 34 / 306$ & 0.94 & 0.63 & 0.80 \\
\hline rs968427 & SFRP1 & 0.39 & $77 / 222 / 188$ & 0.38 & $49 / 165 / 134$ & 0.74 & 0.83 & 0.27 \\
\hline rs921142 & SFRP1 & 0.32 & $53 / 205 / 230$ & 0.31 & $34 / 142 / 168$ & 0.57 & 0.70 & 0.98 \\
\hline
\end{tabular}

DNA methylation. In AzadC-treated cells, 8 genes were up-regulated, whereas 16 were down-regulated (Table 3 ).

\section{Discussion}

In this study we found increased nuclear $\beta$-catenin in osteoblasts from patients with osteoarthritis in comparison with those grown from patients with osteoporotic fractures. $\beta$-Catenin participates in cadherin signaling by binding to the cytoplasmic domain of type I cadherins and linking them to the actin cytoskeleton (Mbalaviele et al., 2006; Nelson and Nusse, 2004). On the other hand, it is a major player in the canonical Wnt pathway. The Wnt pathway regulates the differentiation and activity of bone cells, and particularly of the boneforming cells of the osteoblastic lineage (Williams and Insogna, 2009). On the other hand, Wnt activity has been reported to influence cartilage metabolism and may be involved in the pathogenesis of osteoarthritis (Corr, 2008; Diarra et al., 2007; Lodewyckx and Lories, 2009; Luyten et al., 2009). Although osteoarthritis has been classically understood as a cartilage disorder, important bone changes take place in the vicinity of the joints with osteoarthritis. In fact, several lines of evidence suggest that bone may play more than a merely passive role in the pathogenesis of osteoarthritis (Castaneda et al., 2012). Wnt pathway genes are likely involved in both the bone and cartilage alterations that eventually result in the development of osteoarthritis (Corr, 2008; Kawaguchi, 2009; Lodewyckx and Lories, 2009; Luyten et al., 2009).

We have previously shown a reduced expression of several genes of the Wnt pathway in bone tissue samples and osteoblast cultures from patients with hip fractures, in comparison with samples from patients 358 with osteoarthritis (Velasco et al., 2010). Therefore, it could be speculat- 359 ed that the higher Wnt pathway activity may be involved in the 360 increased bone formation taking place in osteoarthritic joints (causing 361 osteophytes and subchondral bone sclerosis) and perhaps in the differ- 362 ences in bone mass between osteoarthritis and osteoporosis. In the 363 present study we confirmed that nuclear $\beta$-catenin is more abundant 364 in primary osteoblast cultures grown from osteoarthritis samples than 365 in samples from patients suffering a hip fracture. This was not accompa- 366 nied by changes in $\beta$-catenin gene transcription, which is consistent 367 with regulation at the posttranscriptional level. Although we did not 368 confirm increased levels of $\beta$-catenin by western-blotting, these results 369 are in line with studies using gene expression and reporter vectors that 370 showed higher Wnt activity in osteoblasts from patients with osteoar- 371 thritis than in those obtained from fracture cases (Velasco et al., 2010). 372

Genetic association studies have found Wnt pathway genes, and 373 specifically Wnt ligands such as WNT16, to be associated with bone 374 mineral density and wrist fractures (Estrada et al., 2012; Medina- 375 Gomez et al., 2012; Zheng et al., 2012). Therefore, we hypothesized 376 that the differences in Wnt/ß-catenin activity could be related to genetic 377 or epigenetic variants. However, we did not find evidence for genetic 378 differences between both groups of patients regarding three Wnt li- 379 gands (WNT4, WNT10A and WNT16) or a Wnt inhibitor (SFRP1). These 380 negative results of the genetic association analysis should be interpreted 381 in the context of the limitations inherent to our study. Most important, 382 the aim of our study was to explore whether genetic differences contrib- 383 uted to explain the differences in Wnt activity between osteoporotic hip 384

Table 2

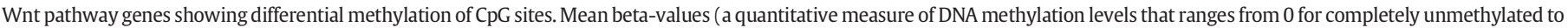

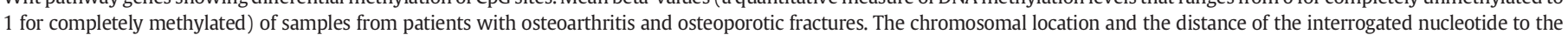
transcription start site (TSS) are shown. FDR, false discovery rate.

\begin{tabular}{|c|c|c|c|c|c|c|}
\hline Gene & CpG location & Distance to TSS & Osteoarthritis (beta) & Fractures (beta) & P-value & FDR \\
\hline FZD10 & 47160656 & 223 & 0.14 & 0.08 & $3.7 \times 10^{-5}$ & 0.0094 \\
\hline CSNK1E & 37044362 & 327 & 0.29 & 0.21 & 0.00036 & 0.0385 \\
\hline TBL1X & 9393597 & 549 & 0.19 & 0.10 & 0.00045 & 0.0385 \\
\hline WNT8A & 137447943 & 365 & 0.54 & 0.61 & 0.00079 & 0.0428 \\
\hline CSNK1A1L & 36577573 & 230 & 0.64 & 0.70 & 0.00083 & 0.0428 \\
\hline SFRP4 & 37922543 & 359 & 0.15 & 0.08 & 0.00176 & 0.0755 \\
\hline
\end{tabular}




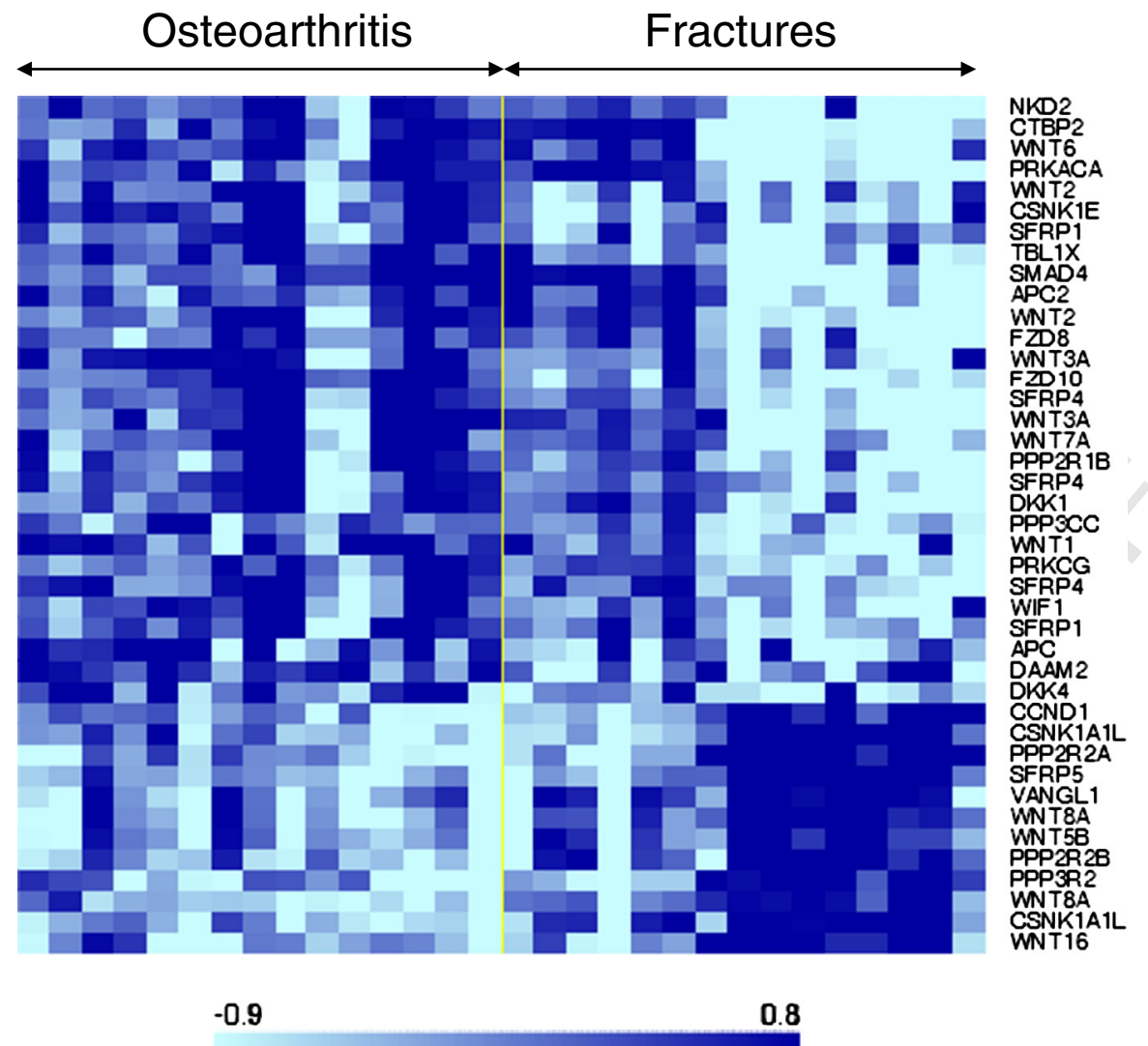

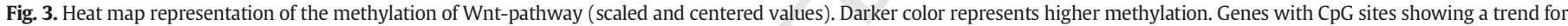
differential methylation between osteoporosis and osteoarthritis (nominal p-values $<0.05$ ) are shown.

fractures and hip osteoarthritis rather than discovery of genes associated with these conditions (reason why comparisons with a healthy control group were not done). This was a moderate-sized study, with limited statistical power. With a type I error of $5 \%$, our study had more than $80 \%$ power to detect disease-associated polymorphisms with odds ratios of 1.4 and 1.6, when the minor allele frequencies are $>0.3$ or $>0.1$, respectively. However, it was underpowered to detect SNPs with smaller odds ratios. For instance, power to detect alleles with 0.3 frequency and odds ratios in the range of 1.1-1.2 would be only 15$41 \%$. In our study sex and age were different in the groups of fractures and osteoarthritis, which reflects the epidemiological differences between these skeletal disorders. A lack of association between the

Table 3

Wnt pathway genes up-regulated and down-regulated by AzadC in MG-63 cells (mean \pm of three experiments).

\begin{tabular}{lcll}
\hline Gene & Fold-increase & Gene & Fold-decrease \\
\hline RAC2 & $18.1 \pm 3.1$ & SFRP1 & $7.6 \pm 0.9$ \\
FZD10 & $12.1 \pm 4.4$ & FZD1 & $6.2 \pm 0.3$ \\
WNT11 & $6.2 \pm 1.6$ & WNT5B & $5.5 \pm 1.2$ \\
PLCB2 & $5.0 \pm 1.0$ & CTNNBIP1 & $4.2 \pm 0.7$ \\
FZD4 & $4.9 \pm 0.6$ & CTNNB1 & $4.1 \pm 0.8$ \\
WNT6 & $3.7 \pm 0.1$ & DKK2 & $4.0 \pm 0.4$ \\
MYC & $3.2 \pm 0.1$ & CSNK2A1 & $3.2 \pm 0.2$ \\
NLK & $2.5 \pm 0.1$ & GSK3B & $3.0 \pm 0.2$ \\
& & CAMK2G & $2.9 \pm 0.2$ \\
& & PPP2R1A & $2.8 \pm 0.4$ \\
& & PLCB3 & $2.7 \pm 0.3$ \\
& & CTBP1 & $2.7 \pm 0.2$ \\
& & CACYBP & $2.3 \pm 0.1$ \\
& & RHOA & $2.3 \pm 0.1$ \\
& & CSNK1A1 & $2.1 \pm 0.1$ \\
& & FOSL1 & $2.1 \pm 0.1$ \\
\hline
\end{tabular}

genotypes and the phenotypes persisted when results were adjusted 397 by age and sex, but the statistical power further decreased under 398 those analysis conditions. We selected genes on the basis of their differ- 399 ential expression, but we cannot exclude the existence of differences in 400 the allelic frequency distributions of other genes in the Wnt pathway. In 401 fact, some investigators reported an association of osteoarthritis with 402 certain polymorphisms of the FRZB gene, which encodes secreted friz- 403 zled related protein 3, another Wnt inhibitor (Loughlin et al., 2004). 404 However, this has not been replicated in other reports, including some 405 recent genome-wide studies (Panoutsopoulou et al., 2011). 406

Since genetic differences did not explain the differences in Wnt ac- 407 tivity, alternative mechanisms not related to DNA sequence might be 408 involved Thus, we hypothesized that epigenetic marks, and specifically 409 cytosine methylation, might underlie the differences in Wnt activity 410 between osteoporosis and osteoarthritis. The methylation of cytosines 411 of CpG dinucleotides is maintained through cell divisions by DNA 412 methyltransferases. Methylation of CpG-rich sequences of the promoter 413 regions tends to inhibit the transcription of genes known to play impor- 414 tant roles in bone formation and bone resorption. On the other hand, the 415 demethylation of those $\mathrm{CpG}$-rich regions is associated with the activa- 416 tion of gene expression (Delgado-Calle et al., 2011, 2012b, 2012c). Little 417 is known about the potential role of $\mathrm{CpG}$ methylation in the pathogen- 418 esis of bone changes in osteoarthritis and other skeletal disorders. How- 419 ever, promoter methylation has been demonstrated to modulate Wnt 420 pathway activity in other normal and neoplastic tissues (Ekstrom 421 et al., 2011; Kocemba et al., 2012). In line with this, in the present 422 study we identified several Wnt-related genes differentially methylated 423 in osteoporosis and osteoarthritis. WNT8A is a Wnt ligand that may be 424 modulated by estrogen and has been associated with alterations of 425 bone development, such as cleft palate (Chiquet et al., 2008). Proteins 426 of the frizzled family, including FZD10 (frizzled family receptor 10), 427 may act as Wnt co-receptors at the cell membranes. On the other 428 
hand, soluble frizzled-related proteins, including SFRP4 (soluble frizzled related protein 4), are secreted and may bind Wnt ligands, thus preventing their interaction with cell membrane receptors (Kawano and Kypta, 2003; Nakanishi et al., 2007; Wang et al., 2006). TBL1X (transducin (beta)-like $1 \mathrm{X}$-linked) encodes a regulatory protein that appears to contribute to the regulation of Wnt target genes ( $\mathrm{Li}$ and Wang, 2008). Casein kinases, including CSNK1E (casein kinase 1 , epsilon), participate in the regulation of a variety of cell functions, and contribute to the signaling cascade initiated by the interaction of Wnt ligands with their receptors (Valle-Perez et al., 2011). The biological role of CSNK1A1L (casein kinase 1, alpha 1-like) gene is unknown.

In theory, those methylation differences could influence Wnt pathway activity, but further studies are needed to confirm this hypothesis, including detailed analysis of methylation at different nucleotides by other procedures such as pyrosequencing. The methylation signatures of Wnt-related genes revealed some rather different patterns, suggesting that the osteoarthritis and fracture groups may be heterogeneous and include patients with somewhat different pathogenetic mechanisms, at least regarding the gene methylation pattern. This is in line with the views of other investigators that used a clinico-epidemiological approach (Herrero-Beaumont et al., 2009). In general, DNA methylation tends to inhibit gene expression, but this is not a universal phenomenon (Hantusch et al., 2007). In fact, we found that AzadC upregulated some Wnt-related genes, but downregulated others. We have previously shown that several genes respond similarly to AZadC treatment in primary osteoblasts and in osteoblastic cell lines (Delgado-Calle et al., 2011, 2012b). Thus, although in the present study we used an osteoblastic cell line to assess the response to AzadC, the results are likely similar in nontransformed osteoblasts. Thus, these experiments support the concept that DNA methylation-dependent mechanisms influence the expression of Wnt pathway genes. However, they do not allow establishing to what extent those changes are the direct consequence of the demethylation of the promoters of those genes, or the result of changes in other regulatory genes upstream in the pathway. For instance, the expression of FZD10 was increased by AzadC, even though its promoter is largely unmethylated in bone samples, with beta-values between 0.08 and 0.14 (see Table 2), and it is even less methylated in cultured osteoblastic cells (unpublished results). Thus, the stimulatory effect of AzadC was likely due to its effect on another regulatory molecule which in turn stimulated FZD10 transcription.

\section{Conclusion}

In conclusion, nuclear $\beta$-catenin levels are higher in osteoblasts form hip osteoarthritis than in osteoblasts from hip fractures. This is in line with previous reports showing higher Wnt pathway activity in osteoarthritis and may be related to the opposite changes in bone mass and bone formation typical of these disorders. However, the difference in Wnt activity is not explained by the allele distribution of common polymorphisms of various Wnt-related genes. On the other hand, despite some heterogeneous patterns, several genes in the Wnt pathway presented differences in methylation. Further studies are needed to elucidate to what extent those epigenetic differences are involved in the differences in Wnt activity.

\section{Founding source}

Supported by grants from Instituto de Salud Carlos III/Fondo de Investigaciones Sanitarias (FIS 06/0034, 09/0539 and PI12/00615). The funding agency has no role in the collection, analysis and interpretation of data; in the writing of the report; or in the decision to submit the article for publication.

\section{Conflicts of interest}

Authors declare that they do not have conflicts of interest.

\section{Acknowledgment}

We acknowledge the excellent technical assistance of Carolina 492 Sañudo and Verónica Mijares. We are grateful to the staff of the Santiago 493 de Compostela Genotyping Center (Centro Español de Genotipado, 494 CEGEN), and particularly to María Torres and Angel Carracedo, for 495 their help in the genotyping study. We also thank technical and scientific 496 supports provided by SGIker (UPV/EHU, MICINN, GV/EJ, ESF).

\section{Appendix A. Supplementary data}

Supplementary data to this article can be found online at http://dx. 499 doi.org/10.1016/j.gene.2013.09.080.

\section{References}

Arcogen Consortium, 2012. Identification of new susceptibility loci for osteoarthritis 502 (arcOGEN): a genome-wide association study. Lancet 380, 815-823.

Arokoski, J.P., Arokoski, M.H., Jurvelin, J.S., Helminen, H.J., Niemitukia, L.H., Kroger, H., 504 2002. Increased bone mineral content and bone size in the femoral neck of men 505 with hip osteoarthritis. Ann. Rheum. Dis. 61, 145-150.

Barrett, J.C., Fry, B., Maller, J., Daly, M.J., 2005. Haploview: analysis and visualization of LD 507 and haplotype maps. Bioinformatics 21, 263-265.

Benjamini, Y., Yekutieli, D., 2005. Quantitative trait loci analysis using the false discovery 509 rate. Genetics $171,783-790$.

odine, P.V., et al., 2005. The Wnt antagonist secreted frizzled-related protein-1 controls 511 osteoblast and osteocyte apoptosis. J. Cell. Biochem. 96, 1212-1230.

Castaneda, S., Roman-Blas, J.A., Largo, R., Herrero-Beaumont, G., 2012. Subchondral bone 513 as a key target for osteoarthritis treatment Biochem. Pharmacol. 83, 315-323. 514

Chaganti, R.K., et al., 2010. Bone mineral density and prevalent osteoarthritis of the hip in 515 older men for the Osteoporotic Fractures in Men (MrOS) Study Group. Osteoporos. 516 Int. $21,1307-1316$. 列 518 enchymal stem cells in craniofacial defects through activation of p38 MAPK. J. Biol. 519 Chem. 282, 30938-30948.

Chiquet, B.T., et al., 2008. Variation in WNT genes is associated with non-syndromic cleft 521 lip with or without cleft palate. Hum. Mol. Genet. 17, 2212-2218. Conde, L., et al., 2006. PupaSuite: finding functional single nucleotide polymorphisms for 523 large-scale genotyping purposes. Nucleic Acids Res. 34, W621-W625.

Corr, M., 2008. Wnt-beta-catenin signaling in the pathogenesis of osteoarthritis. Nat. Clin. 525 Pract. Rheumatol. 4, 550-556.

Delgado-Calle, J., Sanudo, C., Sanchez-Verde, L., Garcia-Renedo, RJ., Arozamena, J, 527 Riancho, J.A., 2011. Epigenetic regulation of alkaline phosphatase in human cells of 528 the osteoblastic lineage. Bone 49, 830-838.

Delgado-Calle, J., Garmilla, P., Riancho, J.A., 2012a. Do epigenetic marks govern bone mass 530 and homeostasis? Curr. Genomics 13, 252-263.

Delgado-Calle, J., et al., 2012b. DNA methylation contributes to the regulation of sclerostin 532 expression in human osteocytes. J. Bone Miner. Res. 27, 926-937.

Delgado-Calle, J., Sanudo, C., Fernandez, A.F., Garcia-Renedo, R., Fraga, M.F., Riancho, J.A., 534 2012c. Role of DNA methylation in the regulation of the RANKL-OPG system in 535 human bone. Epigenetics 7, 83-91.

Dequeker, J., Aerssens, J., Luyten, F.P., 2003. Osteoarthritis and osteoporosis: clinical and 537 research evidence of inverse relationship. Aging Clin. Exp. Res. 15, 426-439. 538

Diarra, D., et al., 2007. Dickkopf-1 is a master regulator of joint remodeling. Nat. Med. 13, 539 156-163.

Ekstrom, E.J., Sherwood, V., Andersson, T., 2011. Methylation and loss of secreted frizzled- 541 related protein 3 enhances melanoma cell migration and invasion. PLoS One 6, 542 e18674.

Estrada, K., et al., 2012. Genome-wide meta-analysis identifies 56 bone mineral density 544

Fraga, M.F., Esteller, M., 2007. Epigenetics and aging: the targets and the marks. Trends 546 Genet. 23, 413-418.

Gaur, T., et al. 2005. Canonical WNT signaling promotes osteogenesis by directly stimulating 548 Runx2 gene expression. J. Biol Chem. 280, 33132-33140. 549

Gordon, M.D., Nusse, R., 2006. Wnt signaling: multiple pathways, multiple receptors, and 550 multiple transcription factors. J. Biol. Chem. 281, 22429-22433.

Hantusch, B. Kalt, R., Krieger, S., Puri, C., Kerjaschki, D., 2007. Sp1/Sp3 and DNA- 552 methylation contribute to basal transcriptional activation of human podoplanin in 553 MG63 versus Saos-2 osteoblastic cells. BMC Mol. Biol. 8, 20.

Hernandez J.L., et al., 2008. Aromatase expression in osteoarthritic and osteoporotic bone. 555 Arthritis Rheum. 58, 1696-1700.

Herrero-Beaumont, G., Roman-Blas, J.A., Castaneda, S., Jimenez, S.A., 2009. Primary osteo- 557 arthritis no longer primary: three subsets with distinct etiological, clinical, and ther- 558 apeutic characteristics. Semin. Arthritis Rheum. 39, 71-80.

6

\section{.}


Jonsson, K.B., Frost, A., Nilsson, O., Ljunghall, S., Ljunggren, O., 1999. Three isolation techniques for primary culture of human osteoblast-like cells: a comparison. Acta Orthop. Scand. 70, 365-373.

Kawaguchi, H., 2009. Regulation of osteoarthritis development by Wnt-beta-catenin signaling through the endochondral ossification process. J. Bone Miner. Res. 24, 8-11.

Kawano, Y., Kypta, R., 2003. Secreted antagonists of the Wnt signalling pathway. J. Cell Sci. 116, 2627-2634.

Kocemba, K.A., et al., 2012. Transcriptional silencing of the Wnt-antagonist DKK1 by promoter methylation is associated with enhanced Wnt signaling in advanced multiple myeloma. PLoS One 7, e30359.

Li, J., Wang, C.Y., 2008. TBL1-TBLR1 and beta-catenin recruit each other to Wnt targetgene promoter for transcription activation and oncogenesis. Nat. Cell Biol. 10, 160-169.

Lodewyckx, L., Lories, R.J., 2009. WNT Signaling in osteoarthritis and osteoporosis: what is the biological significance for the clinician? Curr. Rheumatol. Rep. 11, 23-30.

Loughlin, J., et al., 2004. Functional variants within the secreted frizzled-related protein 3 gene are associated with hip osteoarthritis in females. Proc. Natl. Acad. Sci. U. S. A. 101, 9757-9762.

Luyten, F.P., Tylzanowski, P., Lories, R.J., 2009. Wnt signaling and osteoarthritis. Bone 44, 522-527.

MacDonald, B.T., Tamai, K., He, X., 2009. Wnt/beta-catenin signaling: components, mechanisms, and diseases. Dev. Cell 17, 9-26.

Mbalaviele, G., Shin, C.S., Civitelli, R., 2006. Cell-cell adhesion and signaling through cadherins: connecting bone cells in their microenvironment. J. Bone Miner. Res. 21, 1821-1827.

Medina-Gomez, C., et al., 2012. Meta-analysis of genome-wide scans for total body BMD in children and adults reveals allelic heterogeneity and age-specific effects at the WNT16 locus. PLoS Genet. 8, e1002718.

Nakanishi, R., et al., 2007. Osteoblast-targeted expression of Sfrp4 in mice results in low bone mass. J. Bone Miner. Res.

Nelson, W.J., Nusse, R., 2004. Convergence of Wnt, $\beta$-catenin, and cadherin pathways. Science 303, 1483-1487.

Panoutsopoulou, K., et al., 2011. Insights into the genetic architecture of osteoarthritis from stage 1 of the arcOGEN study. Ann. Rheum. Dis. 70, 864-867.
Piters, E., Boudin, E., Van Hul, W., 2008. Wnt signaling: a win for bone. Arch. Biochem. 594 Biophys. 473, 112-116.

Purcell, S., et al., 2007. PLINK: a tool set for whole-genome association and population- 596 based linkage analyses. Am. J. Hum. Genet. 81, 559-575. 597

Ralston, S.H., 2010. Osteoporosis as an hereditary disease. Clin. Rev. Bone Miner. Metab. 8, 598 $68-76$.

Riancho, J.A., et al., 2011. Wnt receptors, bone mass, and fractures: gene-wide association 600 analysis of LRP5 and LRP6 polymorphisms with replication. Eur. J. Endocrinol. 164, 601 123-131.

Richards, J.B., et al., 2009. Collaborative meta-analysis: associations of 150 candidate 603 genes with osteoporosis and osteoporotic fracture. Ann. Intern. Med. 151, 528-537. 604

Styrkarsdottir, U., et al., 2009. New sequence variants associated with bone mineral density. 605 Nat. Genet. 41, 15-17.

Valdes, A.M., et al., 2007. Sex and ethnic differences in the association of ASPN, CALM1, 607 COL2A1, COMP, and FRZB with genetic susceptibility to osteoarthritis of the knee. 608 Arthritis Rheum. 56, 137-146.

Valero, C., et al., 2011. Relationship of sclerostin and secreted frizzled protein polymor- 610 phisms with bone mineral density: an association study with replication in postmen- 611 opausal women. Menopause 18, 802-807.

Valle-Perez, B., Arques, O., Vinyoles, M., de Herreros, A.G., Dunach, M., 2011. Coordinated 613 action of CK1 isoforms in canonical Wnt signaling. Mol. Cell. Biol. 31, 2877-2888. 614

van Amerongen, R., Nusse, R., 2009. Towards an integrated view of Wnt signaling in 615 development. Development 136, 3205-3214.

Velasco, J., et al., 2010. Wnt pathway genes in osteoporosis and osteoarthritis: differential 617 expression and genetic association study. Osteoporos. Int. 21, 109-118 618

Wang, H.Y., Liu, T., Malbon, C.C., 2006. Structure-function analysis of Frizzleds. Cell. Signal. 619 $18,934-941$.

Williams, B.O., Insogna, K.L., 2009. Where Wnts went: the exploding field of Lrp5 and 621 Lrp6 signaling in bone. J. Bone Miner. Res. 24, 171-178.

Yao, W., Cheng, Z., Shahnazari, M., Dai, W., Johnson, M.L., Lane, N.E., 2010. Overexpression 623 of secreted frizzled-related protein 1 inhibits bone formation and attenuates parathy- 624 roid hormone bone anabolic effects. J. Bone Miner. Res. 25, 190-199. 625

Zheng, H.F., et al., 2012. WNT16 influences bone mineral density, cortical bone thickness, 626 bone strength, and osteoporotic fracture risk. PLoS Genet. 8, e1002745. 627 